\title{
Dopant Diffusion and Current-Voltage Studies on Epitaxial InP Codoped with $\mathrm{Ru}$ and $\mathrm{Re}^{*}$
}

\author{
D. SÖDERSTRÖM,,$^{1,3}$ S. LOURDUDOSS, ${ }^{1}$ A. DADGAR, ${ }^{2}$ O. STENZEL, ${ }^{2}$ D. \\ BIMBERG ${ }^{2}$ and H. SCHUMANN ${ }^{2}$ \\ 1.-Royal Institute of Technology, Laboratory of Semiconductor Materials, Department of Electron- \\ ics, Electrum 229. S-164 40 Kista, Sweden. 2.-Technische Universität Berlin, Hardenbergstr. 36, \\ 10623 Berlin, Germany. 3.—e-mail: david@ele.kth.se
}

The following table was inadvertantly omitted when this article was originally printed in the August 2001 issue of the Journal of Electronic Materials, Vol. 30, No. 8, pp. 972-976.

\begin{tabular}{|c|c|c|c|c|}
\hline \multicolumn{5}{|c|}{$\begin{array}{l}\text { Table I. Ru and Fe Concentrations } \\
\text { in the Samples in the Investigation }\end{array}$} \\
\hline Identity & $\begin{array}{l}\text { SI- } \\
\text { Layer }\end{array}$ & $\underset{\left(\mathbf{c m}^{-3}\right)}{\mathbf{R u}}$ & $\begin{array}{c}\mathbf{F e} \\
\left(\mathrm{cm}^{-3}\right)\end{array}$ & $\begin{array}{c}\text { Thickness } \\
(\mu \mathrm{m})\end{array}$ \\
\hline \multicolumn{5}{|l|}{ n(sub)-SI-n } \\
\hline $725 \mathrm{D} 1$ & InP:Ru & $3.9 \times 10^{17}$ & - & 3.5 \\
\hline $732 \mathrm{D} 2$ & InP:Ru,Fe & $1.9 \times 10^{17}$ & $4.9 \times 10^{17}$ & 4.1 \\
\hline \multirow{2}{*}{\multicolumn{5}{|c|}{ p(sub)-SI-p }} \\
\hline & & & & \\
\hline $725 \mathrm{~B} 2$ & InP:Ru & $4.0 \times 10^{17}$ & - & 3.5 \\
\hline $732 \mathrm{~A} 2$ & InP:Ru,Fe & $1.9 \times 10^{17}$ & $4.9 \times 10^{17}$ & 4.2 \\
\hline $735 \mathrm{C} 1$ & InP:Ru,Fe & $2.7 \times 10^{17}$ & $4.5 \times 10^{17}$ & 4.5 \\
\hline
\end{tabular}

